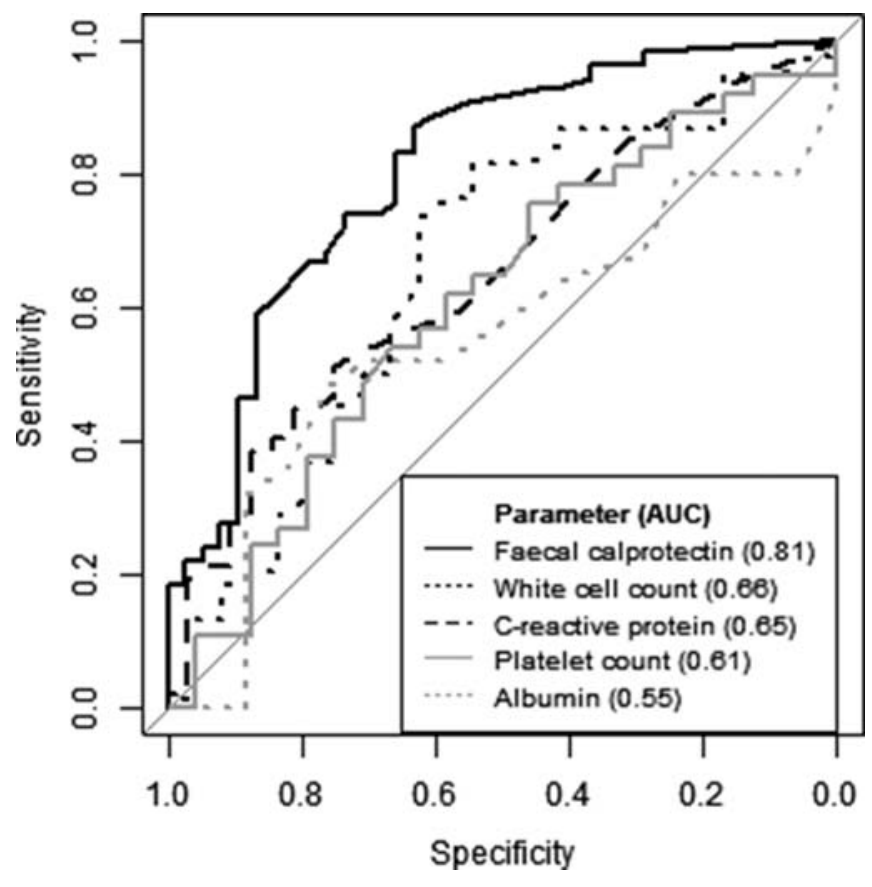

Abstract PTU-081 Figure 1

45 years (IQR 32-56); median follow-up 34 months (IQR 2544). The median (IQR) FC was $80 \mu \mathrm{g} / \mathrm{g}(20-142)$ where SBMRI demonstrated no active ileal disease (simple MRI score $=0, \mathrm{n}=$ 38), $198 \mu \mathrm{g} / \mathrm{g}(120-444)$ for mild to moderate $(1-6, \mathrm{n}=30)$ and $398 \mu / \mathrm{g}(168-771)$ for severe disease $(>6, \mathrm{n}=24)(\mathrm{p}<$ 0.001). ROC analysis showed an AUC of $0.81(0.72-0.90)$ for FC which was significantly higher than for CRP (0.65 [0.530.77], $\mathrm{p}=0.020$ ) (Figure 1).

Conclusion FC correlates closely with SBMRI findings in ileal Crohn's disease and outperforms other laboratory tests. In future, following validation, we will derive clinical useful MRI and FC cut-offs that predicate on important patient outcomes. Disclosure of Interest None Declared.

\section{PTU-082 PAEDIATRIC INFLAMMATORY BOWEL DISEASE UNCLASSIFIED IN SCOTLAND: INCIDENCE AND NATURAL HISTORY}

${ }^{1} \mathrm{FL}$ Cameron*, ${ }^{1} \mathrm{P}$ Henderson, ${ }^{2}$ RK Russell, ${ }^{1} \mathrm{DC}$ Wilson. ${ }^{1}$ Child Life and Health, University of Edinburgh, Edinburgh, UK; ${ }^{2}$ Paediatric Gastroenterology, Royal Hospital for Sick Children, Glasgow, UK

\subsection{6/gutjnl-2014-307263.156}

Introduction Inflammatory Bowel Disease Unclassified (IBDU) accounts for $\sim 10 \%$ of paediatric IBD (PIBD). The natural history of IBDU includes potential evolution to Crohn's disease (CD) or ulcerative colitis (UC). Few epidemiologically robust studies of IBDU exist so we aimed to describe the incidence and the natural history of paediatric IBDU in a population-based cohort.

Methods Incidence of IBDU was collected over a 10-year period (01/03-12/12) from the two largest Scottish paediatric gastroenterology networks (serving $74.4 \%$ of Scottish population $<16$ years). Demographics, diagnostic investigation and follow-up data were obtained until study end (31.10.13; unless prior transition or emigration) to ascertain reclassification of IBD subtype, clinical progress and outcome at last follow up. Incidence rates and trends were calculated using publicly available population data and statistics generated using Poisson regression.

Results 65 patients were IBDU (57\% male) at diagnosis with a median age of 11.3yrs (range 2.6-15.9). The age adjusted incidence of IBDU was $0.65 / 100,000 / \mathrm{yr}(95 \%$ CI $0.42-0.97)$ in the 5 -year epoch 2003-2007 and 1.14/100,000/yr (95\% CI 0.811.56) for 2008-2012, a non-significant increase $(\mathrm{p}=0.068)$.

All patients had colonoscopy (74\% ileal intubation), 62 (95\%) had an upper GI endoscopy; remaining 3 had small bowel imaging. 61 (94\%) had radiological imaging, 44 (68\%) had a barium meal and follow through with 15 (23\%) having MR enterography. At diagnosis, $53(82 \%)$ had a pancolitis, $4(6 \%)$ had disease distal to the hepatic flexure, $5(8 \%)$ had disease distal to the splenic flexure and 3 (4\%) had proctitis. 37 (57\%) had mild disease (defined as mild infrequent relapses) while 7 (11\%) had severe chronically active disease with $5(8 \%)$ requiring Infliximab. Median follow-up was 3.1 yrs (range 0.4-6.8). 16 (25\%) had their diagnosis changed (all after endoscopic re-evaluation) after a median of 1.6yrs (range $0.6-5.7$ ), to CD in 11 and UC in $5 ; 10(15 \%)$ remained IBDU. $3(4 \%)$ required surgery and had colectomy and end ileostomy, $2(66 \%)$ had diagnosis changed to UC prior to surgery whilst the other remained IBDU and reclassified to CD later.

Conclusion In the first ever UK population-based epidemiological study of IBDU, the incidence of IBDU demonstrated a nonsignificant trend to rise during the 10 year study period. $25 \%$ of patients had their diagnosis changed after endoscopic re-evaluation, more than previously reported by systematic review (Prenzel and Uhlig JCC 2009) after median follow up of 1.6 years. Most cases had inactive/mild disease activity; a minority remained IBDU despite re-assessment.

Disclosure of Interest None Declared.

\section{PTU-083 USE OF POLYMERASE CHAIN REACTION TO DETECT MUCOSAL CYTOMEGALOVIRUS INFECTION IN PATIENTS WITH ACUTE ULCERATIVE COLITIS}

${ }^{1}$ GSZ Tun*, ${ }^{2} \mathrm{~A}$ Wright, ${ }^{2} \mathrm{~K}$ Robinson, ${ }^{2} \mathrm{R}$ Sidhu, ${ }^{2} \mathrm{~A}$ Hopper, ${ }^{2} \mathrm{DS}$ Sanders, ${ }^{2} \mathrm{M}$ McAlindon, ${ }^{3} \mathrm{M}$ Raza, ${ }^{2} \mathrm{~A}$ Lobo. ${ }^{1}$ Medicine, Sheffield Teaching Hospitals NHS Trust, Sheffield, UK; ${ }^{2}$ Gastroenterology, Sheffield Teaching Hospitals NHS Trust, Sheffield, UK; ${ }^{3}$ Virology, Sheffield Teaching Hospitals NHS Trust, Sheffield, UK

\subsection{6/gutjnl-2014-307263.157}

Introduction Mucosal CMV infection may complicate acute ulcerative colitis (UC), though the significance remains uncertain. The European Crohn's and Colitis Organisation recommend tissue PCR or immunohistochemistry for CMV detection. The aim of this study was to review experience of assessing patients attending a single unit with acute UC for CMV infection.

Methods A policy of biopsy for CMV infection was adopted for people with acute severe UC admitted to hospital ( $\mathrm{n}=37$ ) or deteriorating symptoms as an out-patient $(\mathrm{n}=8)$ from 20112013. Clinical severity was measured by Mayo score and endoscopic activity by Baron score. Biopsies were assessed for CMV viral DNA by realtime PCR. Serum IgG and IgM antibodies to CMV were measured by chemiluminescence.

Results Biopsies were obtained from 45 patients with UC. 13/45 (28.9\%) were positive for CMV DNA (median titre 34900; range 776-1540000 copies/ml). 9/13 (69.2\%) CMV PCR positive (+) patients were steroid refractory compared to $14 / 32$ (43.8\%) CMV PCR negative $(-) \mathrm{p}=0.12$. Median Day 3 Mayo scores were 8 for the CMV+ group and 6 for the CMV- group $(p=0.59)$. Of IPs biopsied up to Day 1 of IV steroids (range $-4-1$ ), 6/22 were CMV 
+ and from Day 2 (median 6; 2-19), 5/20 were CMV+ (p = 0.96). Median cumulative steroid dose prior to biopsy was $0.28 \mathrm{~g}$ prednisolone equivalent for $\mathrm{CMV}+$ patients and $0.39 \mathrm{~g}$ for CMVpatients $(\mathrm{p}=0.51) .3 / 13 \mathrm{CMV}+$ patients had had prednisolone $\geq 10 \mathrm{mg} /$ day for $>14$ days prior to biopsy and 11/32 CMVpatients $(p=0.46)$. No biopsies contained typical histological features of CMV infection. Mayo and Baron scores did not differ between CMV+ and CMV- patients. Mucosal CMV DNA titre in $\mathrm{CMV}+$ patients did not correlate with Baron or Mayo scores. Of the mucosal CMV+ patients, $8 / 8$ tested $(100 \%)$ were anti-CMV IgG positive with $0 / 7$ IgM positive. Peripheral blood CMV PCR was positive in 10/13 in the biopsy PCR + group and 0/7 in those biopsy PCR- (sensitivity 60\%, specificity 100\%). 12/13 CMV PCR + patients were treated with ganciclovir. 11/13 (84.6\%) achieved remission. 2/13 (15.3\%) CMV+ and 2/32 (6.2\%) CMV- required colectomy $(\mathrm{p}=0.33)$. All 6 patients treated concomitantly with Infliximab and ganciclovir responded fully.

Conclusion PCR of mucosal biopsies detects CMV infection due to viral reactivation in almost a third of patients with deteriorating or acute severe UC. No relationship was demonstrated between CMV infection and disease severity, response to treatment or prior steroid use. Treatment with anti-TNF agents was administered safely in combination with anti-viral drugs. Disclosure of Interest None Declared.

\section{PTU-084 PREVENTION OF OPPORTUNISTIC INFECTIONS IN PATIENTS ON BIOLOGICAL AGENTS FOR MANAGEMENT OF INFLAMMATORY BOWEL DISEASE}

H Gordon*, A Steel. Gastroenterology, Chelsea and Westminster Hospital, London, UK

\subsection{6/gutjnl-2014-307263.158}

Introduction Patients with inflammatory bowel disease are at increased risk of infection; this is especially true of the $20 \%$ on biological agents. ECCO guidelines recommend the following vaccines:

Influenza (annual), Pneumococcal, Hepatitis B, Varicella, HPV (women under 26).

The guidelines also highlight the need to exclude latent TB; local policy is to perform an interferon gamma release assay.

Within the UK vaccination services are provided by primary care.

Methods The measures taken to prevent opportunistic infection in patients prescribed anti-TNFs for IBD at Chelsea and Westminster Hospital in 2013 were audited against the ECCO OI Guidelines.

The following were retrieved from electronic records: age, sex, anti TNF prescribed, pneumococcal antibodies, hepatitis B core and surface antibodies, varicella $\operatorname{IgG}$, Elispot ${ }^{\circledR}$.

Attempts were made to retrieve vaccination history from General Practice.

Results 60 patients were prescribed infliximab and 15 patients were prescribed adalimumab. 46 GPs were able to provide vaccination history.

Influenza: $50 \%(23 / 46)$ patients received vaccination against influenza within the past year.

Pneumococcus: $55 \%(47 / 85)$ patients demonstrated immunity. $6 \%(5 / 85)$ were not immune and the remainder were not tested. The vaccination history of 26 patients who were not immune or not tested was retrieved. 27\% (7/26) had since been vaccinated.

Hepatitis B: No patients were core Ab positive. Surface Ab levels demonstrated immunity in 7\% (6/85). 53\% (45/85) were not immune, and the remainder were not tested. Vaccination history of 44 patients who were not immune or not tested was retrieved. Of these, $25 \%(11 / 44)$ had since been vaccinated.

HPV: 4 patients were women under 26 years old. $25 \%(1 / 4)$ had confirmed HPV vaccination.

Varicella: $21 \%(18 / 85)$ patients demonstrated immunity to varicella. $2 \% 2 / 85$ were not immune.

Elispot: $65 \%(55 / 85)$ patients had a nonreactive assay. 1\% (1/ 85 ) had a positive result and the remainder were not tested.

Conclusion The standards set out by ECCO to protect patients from opportunistic infection are not being met.

Problems obtaining accurate vaccination history from GP records include incorrect surgery details, lack of availability of staff able to review records and incomplete records. HPV vaccination usually takes place at school and is not routinely recorded by primary care.

Potential service improvements include provision of vaccines at clinic, improved patient education regarding the importance of vaccination and a check list to review bloods at first anti-TNF prescription.

\section{REFERENCE}

1 Rahier JF, Ben-Horin S, Chowers $Y$, Conlon C, De Munter P, D'Haens G, Domènech E, Eliakim R, Eser A, Frater J, et al. European evidence-based Consensus on the prevention, diagnosis and management of opportunistic infections in inflammatory bowel disease. J Crohns Colitis 2009;3:47-91

Disclosure of Interest None Declared.

\section{PTU-085 DID THE 2010 NICE GUIDELINES IMPACT ON BIOLOGICS PRESCRIPTION?}

${ }^{1}$ H Steed ${ }^{*},{ }^{2}$ A Townsend, ${ }^{1}$ A Rowlinson, ${ }^{1} \mathrm{~F}$ Leslie. 'Gastroenterology, University Hospital North Staffordshire, UK; ${ }^{2}$ Medical School, Keele University, Stoke-on-Trent, UK

\subsection{6/gutjnl-2014-307263.159}

Introduction In 2010 NICE updated their guidelines on the prescription of anti-tumour necrosis factor alpha (a-TNF) for Crohn's disease (CD) to include Adalimumab as a treatment for patients with severe active CD. As a therapeutic option it provided an attractive alternative, both to units, struggling for infusion capacity and limited specialist nurse input, and to patients, as a time-saving, home delivered and administered option.

Methods A retrospective review of inflammatory bowel disease patients at University Hospital North Staffordshire up until December 2012.

Results Of 3600 inflammatory bowel disease 117 patients are on a-TNF. A further 47 patients have had, but are no longer on, a-TNF. 37 patients had their original a-TNF switched during their disease course (21\%). 25 patients (68\% of all switches) had their a-TNF switched for non-clinical reasons ("including patient choice and unit preference). All of these were from infliximab to adalimumab, 8 had to be switched back to infliximab.

\begin{tabular}{ll} 
Abstract PTU-085 Table 1 & \\
\hline Year & No of non-clinical switches \\
\hline 2008 & 3 \\
2009 & 0 \\
2010 & 15 \\
2011 & 3 \\
2012 & 4 \\
\hline
\end{tabular}

